\title{
Krasnoselskii-type fixed point theorem in ordered Banach spaces and application to integral equations
}

\section{Théorème du point fixe de type Krasnoselskii dans les espaces de Banach ordonnés et application aux équations intégrales}

\author{
Abdelhamid Benmezaï \\ Faculty of Mathematics, USTHB, Algiers, Algeria \\ aehbenmezai@gmail.com
}

ABSTRACT. In this paper, we present a variant of Krasnoselskii's fixed point theorem in the case of ordered Banach spaces, where the order is generated by a normal and minihedral cone. In such a structure, there is a possibility to give a new sence to the concept of contraction.

AMS Subject Classification. 47H10; 47H07, 47A10; 45G10.

KEYWORDS. Fixed point theory, Cones, Positive operators.

\section{Introduction}

Many nonlinear problems arising from applications in most domains of natural sciences and physics are formulated as fixed point equation having the form

$$
x=A x+B x, x \in M
$$

where $M$ is a closed convex set (a ball of radius $r$ in practice), of a functional Banach space. Especially, many nonlinear integral equations are written in that form, see $[5,9,10,11,12]$.

In 1958, Krasnoselskii established the first and the most famous fixed-point principal solving Equation (1.1), see [14]. His result states that if $A$ is a contraction, $B(M)$ is relatively compact and $M$ is left invariant by the operators sum $A+B$, then Equation (1.1) admits a solution in $M$.

Since then, the existence of fixed points for the sum of two operators has attracted tremendous interest and many forms and improvements of Krasnoselskii's fixed point theorem, have been established in the literature by modifying the above assumptions; see, for example, $[1,2,3,4,5,6,9,10,11,15,17,18$, $19,21]$. Often, the application of the obtained results is guaranteed.

The main goal of this work is to adapt the Krasnoselskii's fixed point principal to the case of ordered Banach spaces. More precisely, we consider in this article, the case of ordered Banach spaces where the order is generated by a minihedral and normal cone. In such a structure, the absolute value of a vector has a sense and this offers the possibility to give a new sense to the concept of contraction, see Condition 3 in Theorem 3.2 .

The paper is organized as follows: in Section 2 we recall some definitions and basic facts related to cones, positive operator and elementary spectral theory, which will be needed for the proof of the main result of this article. In Section 3, we present an adapted version of Krasnoselskii's fixed theorem to the case of ordered Banach spaces and the corresponding alternative. The last section is devoted for the study of existence of solutions to a nonlinear integral equation posed on the whole real line. 


\section{Abstract background}

The main goal of this section is to recall some basic facts on cones, ordered Banach spaces and the elementary spectral theory. We recall also the classical Schauder's fixed theorem and the corresponding alternative, commonly know as Schaefer's fixed point theorem. All are needed for the statement of the main theorem of this work and its proof. Let $X$ be a real Banach space endowed with a norm $\|\cdot\|$.

Definition 2.1. A closed convex nonempty subset $C$ of $X$ is said to be a cone in $E$ if $(t C) \subset C$ for all $t \geq 0$ and $C \cap(-C)=\left\{0_{X}\right\}$.

It is well known that a cone $C$ induces a partial order in the Banach space $X$. We write for all $x, y \in$ $X: x \preceq_{C} y$ (or $y \succeq_{C} x$ ) if $y-x \in C$ and $x \prec_{C} y$ (or $y \succ_{C} x$ ) if $y-x \in C \backslash\left\{0_{X}\right\}$. Thus, the vectors lying in $C \backslash\left\{0_{X}\right\}$ are said to be positive.

Definition 2.2. Let $\Omega$ be a nonempty set in $X$. Then

a) $u \in X$ is said to be an upper bound of $\Omega$ if $v \preceq u$ for all $v \in \Omega$;

b) $u \in X$ is said to be a lower bound of $\Omega$ if $v \succeq u$ for all $v \in \Omega$;

c) $u \in X$ is said to be the least upper bound of $\Omega$ and we write $u=\sup \Omega$, if $u$ is an upper bound of $\Omega$ and $v \preceq w$ for all $v \in \Omega$ implies $u \preceq w$;

d) $u \in E$ is said to be the greatest lower bound of $\Omega$ and we write $u=\inf \Omega$, if $u$ is a lower bound of $\Omega$ and $v \succeq w$ for all $v \in \Omega$ implies $u \succeq w$.

Definition 2.3. Let $C$ be a cone in $X$. Then

a) $C$ is normal if there is a positive constant $n_{C}$ such that for all $u, v \in E, 0_{E} \preceq_{C} u \preceq_{C} v$ implies $\|u\| \leq n_{C}\|v\|$;

b) $C$ is minihedral if $\sup (x, y)$ exists for all $x, y \in X$.

Remark 2.4. Notice that if a cone $C$ is minihedral then $\inf (x, y)$ exists for all $x, y \in X$. Moreover, we have $\inf (x, y)=-\sup (-x,-y)$.

Remark 2.5. It is well known that if $C$ is a minihedral cone inducing the order $\preceq_{C}$ on $X$, then $\left(X, \preceq_{C}\right)$ is a Riesz space or a Banach lattice in the sence given in [16]..

Definition 2.6. Let $C$ be a minihedral cone in $X$ inducing the order $\preceq_{C}$ on $X$. For $x \in X$, we define the positive part, the negative part and the absolute value of the vector $x$ respectively by

$$
x^{+}=\sup (x, 0), \quad x^{-}=\sup (-x, 0) \quad \text { and } \quad|x|=x^{+}+x^{-} .
$$

Proposition 2.7. ([16]) Let $C$ be a minihedral cone in E inducing the order $\preceq_{C}$ on $E$. Then the absolute value define then a self-mapping on $E$ and it has the following properties:

i) $|x| \succeq_{C} 0_{X}$ for all $x \in X$,

ii) $|x|=0_{X} \Rightarrow x=0_{X}$,

iii) $|t x|=|t||x|$ for all $x \in X$ and $t \in \mathbb{R}$, 
iv) $|x+y| \preceq_{C}|x|+|y|$ for all $x, y \in X$,

v) ||$x|-| y|| \preceq_{C}|x-y|$ for all $x, y \in X$.

Proposition 2.8. Let $C$ be a minihedral cone in $X$, then the following assertions are equivalents.

i) The mapping $|\cdot|: X \rightarrow C$ is continuous.

ii) The mapping $|\cdot|: X \rightarrow C$ is continuous at $0_{X}$.

iii) There exists $\eta>0$ such that $\||u|\| \leq \eta\|u\|$ for all $u \in X$.

Proof. The equivalence between i) and ii) is due to the inequality in v) of Proposition 2.7. It is easy to see that iii) implies ii) and, hence let us prove that ii) implies iii). Let $\epsilon_{0}>0$, there is $\delta_{0}>0$ such that for all $u \in X,\|u\| \leq \delta_{0}$ implies $\||u|\| \leq \epsilon_{0}$. Therefore, for all $u \in X$ with $u \neq 0_{X}$, we have

$$
\frac{\delta_{0}}{\|u\|}\||u|\|=\left\|\frac{\delta_{0} u}{\|u\|} \mid\right\| \leq \epsilon_{0}
$$

leading to

$$
\||u|\| \leq \eta\|u\| \text { for all } u \in X
$$

with $\eta=\epsilon_{0} / \delta_{0}$.

Remark 2.9. It follows from Proposition 2.8 that the mapping $|\cdot|: X \rightarrow C$ is continuous if and only if $\sup _{\|u\|=1}\||u|\|<\infty$.

Definition 2.10. Let $C$ be a cone in $X$. A mapping $L \in \mathcal{L}(X)$ is said to be positive, if $L(C) \subset C$.

Throughout, $\mathcal{L}_{C}(X)$ will denote the subset in $\mathcal{L}(X)$ of all positive mapping relatively to the cone $C$.

For detailled presentations on cones and positivity we refer the reader to [8] and [13]. The reader will observe that the definition of the minihedrality given here is that of [8]. In [13], a cone $C$ is said to be minihedral if $\sup (x, y)$ exists for all pair $(x, y) \in X^{2}$ having an upper bounded. To ensure the existence of $\sup (x, y)$ for all $x, y \in X$ when such is the definition of the minihedrality, one may assume that the cone $C$ is generating (i.e. $X=C-C$ ). Indeed, for all $x, y \in X$ there exist $x_{1}, x_{2}, y_{1}, y_{2} \in C$ such that $x=x_{1}-x_{2}$ and $y=y_{1}-y_{2}$. Therefore, we have $x \preceq_{C} x_{1}+y_{1}$ and $y \preceq_{C} x_{1}+y_{1}$.

In all this work, we use the following notations: for $L \in \mathcal{L}(X), C V(L)$ denotes the set of all characteristic values of $L$. The spectral radius of $L$, is defined to be

$$
r(L)=\left\{\begin{array}{lc}
\inf \left\{|\mu|^{-1}:\right. & \mu \in C V(L)\}, \\
0, & \text { if } C V(L) \neq \emptyset,
\end{array}\right.
$$

and we have by the Gelfand formula

$$
r(L)=\lim _{n \rightarrow \infty}\left\|L^{n}\right\|^{1 / n} .
$$

For $\mu \notin C V(L), R(\mu, L)=(I-\mu L)^{-1}$ is the resolvent mapping associated with $L$ and we have for all $\mu \in \mathbb{R}$ with $|\mu|<1 / r(L)$,

$$
R(\mu, L)=(I-\mu L)^{-1}=\sum_{n=0}^{\infty} \mu^{n} L^{n} .
$$


Notice that if $C$ is a cone in $X$ we have from (2.1) that $R(\mu, L) \in \mathcal{L}_{C}(X)$ for all $L \in \mathcal{L}_{C}(E)$ and all $\mu \in(0,1 / r(L))$.

A detailled presentation on the elementary spectral theory is found in Yoshida's book [23].

We end this section by the following two classical fixed point theorems.

Theorem 2.11 (Schauder 1930 [20]). Let $M$ be a closed convex nonempty subset of $X$ and let $S: M \rightarrow$ $M$ be a compact mapping. Then $S$ has a fixed point.

Theorem 2.12 (Scheafer 1955 [20]). Let $S: X \rightarrow X$ be a completely continuous mapping. Then either

1. S has a fixed point, or

2. the set $\{x \in X: x=\lambda S(x) \lambda \in(0,1)\}$ is unbounded.

\section{A variant of Krasnoselskii's fixed point Theorem}

In all this section, we let $E$ be a real Banach space, $K$ be a cone in $E$ and we set for $L \in \mathcal{L}_{K}(E)$,

$$
\lambda_{L, K}^{-}= \begin{cases}\inf \Lambda_{L, K}^{-}, & \text {if } \Lambda_{L, K}^{-} \neq \emptyset \\ +\infty, & \text { if } \Lambda_{L, K}^{-}=\emptyset\end{cases}
$$

where

$$
\Lambda_{L, K}^{-}=\left\{\lambda>0: \quad \exists u \succ_{K} 0_{E} \text { such that } \lambda L u \succeq_{K} u\right\}
$$

We begin this section by the following technical Lemma.

Lemma 3.1. For all $L \in \mathcal{L}_{K}(E), \lambda_{L, K}^{-} \geq 1 / r(L)$.

Proof. The cases where $\Lambda_{L, K}^{-}=\emptyset$ or $r(L)=0$ are obvious, so let us prove the lemma in the case where $\Lambda_{L, K}^{-} \neq \emptyset$ and $r(L)>0$. Let $\lambda \in \Lambda_{L, K}^{-}, u \succ_{K} 0_{E}$ such that $\lambda L u \succeq_{K} u$. By the contrary, suppose that $\lambda>1 / r(L)$ and set $v=-(I-\lambda L)(u)$. Since $\lambda>1 / r(L),(I-\lambda L)$ is invertible and $(I-\lambda L)^{-1}=\sum_{n \in \mathbb{N}} \lambda^{n} L^{n}$. Thus, we have the contradiction

$$
0_{E} \prec_{K} u=(I-\lambda L)^{-1}(v)=-\sum_{n \in \mathbb{N}} \lambda^{n} L^{n}(v) \preceq_{K} 0_{E} .
$$

The lemma is proved.

The following theorem is the main result of this paper. It provides a variant of Krasnoselskii's theorem in the ordered Banach space $E$.

Theorem 3.2. Assume that the cone $K$ is normal and minihedral and the mapping $|\cdot|: E \rightarrow K$ is continuous. If $\Omega$ is a closed convex nonempty subset of $E$ and the mappings $S, T: E \rightarrow E$ are such that

1. $S u+T v \in \Omega$ for all $u, v \in \Omega$,

2. $S$ is compact and 
3. there is $L$ in $\mathcal{L}_{K}(E)$ and $c \geq 0$ such that $\operatorname{cr}(L)<1$ and

$$
|T u-T v| \preceq_{K} c L(|u-v|) \text { for all } u, v \in E .
$$

Then $S+T$ has a fixed point in $\Omega$.

Proof. Let $n_{K}$ be the constant of normality of $K$ and $\eta=\sup _{\|u\|=1}\||u|\|$.

The proof is divided into three steps.

Step 1. In this step, we prove that for all $v \in E$ the equation

$$
u=T u+v
$$

admits a unique solution in $E$.

The case $c=0$ is obvious, so we suppose that $c>0$.

Uniqueness. If $u_{1}$ and $u_{2}$ are two solutions to (3.1), then $w=\left|u_{1}-u_{2}\right| \succ_{K} 0_{E}$ and satisfies

$$
w=\left|u_{1}-u_{2}\right|=\left|T u_{1}-T u_{2}\right| \preceq_{K} c L\left(\left|u_{1}-u_{2}\right|\right)=c L w .
$$

Hence, $c \in \Lambda_{L, K}^{-} \neq \emptyset$ and $r\left(L_{F}\right)>0$. Indeed, we have by induction

$$
L^{n} w \succeq_{K} \frac{1}{c^{n}} w
$$

and then the normality of the cone $K$ leads to

$$
\left\|L^{n}\right\|\|w\| \geq\left\|L^{n} w\right\|=\left\|L^{n} w\right\| \geq \frac{1}{n_{K} c^{n}}\|w\| .
$$

From which we see that

$$
r(L)=\lim _{n \rightarrow+\infty} \sqrt[n]{\left\|L^{n}\right\|} \geq \frac{1}{c}>0 .
$$

Therefore, this together with Lemma 3.1 lead to the contradiction

$$
\lambda_{L, K}^{-}>1 / r(L)>c \geq \inf \left\{\lambda \geq 0: \exists u \succ_{K} 0_{E} \text { such that } \lambda L u \succeq_{K} u\right\}=\lambda_{L, K}^{-} .
$$

The uniqueness is proved.

Existence. Let $u_{0} \in E$ and consider the sequence $\left(u_{n}\right)$ defined by $u_{n}=T u_{n-1}+v$. We have then for all $n \geq 1$,

$$
\left|u_{n+1}-u_{n}\right|=\left|T\left(u_{n}\right)-T\left(u_{n-1}\right)\right| \preceq_{K} c L\left(\left|u_{n}-u_{n-1}\right|\right) .
$$

Since the operator $L$ is increasing, we obtain

$$
\left|u_{n+1}-u_{n}\right| \preceq_{K} c^{n} L^{n}(w),
$$

where $w=\left|u_{1}-u_{0}\right|$

Therefore, if $m, n$ are two integers with $m>n \geq 1$, then

$$
\begin{aligned}
\left|u_{m}-u_{n}\right| & \preceq_{K}\left|u_{m}-u_{m-1}\right|+\left|u_{m-1}-u_{m-2}\right|+\ldots+\left|u_{n+1}-u_{n}\right| \\
& \preceq_{K} c^{m-1} L^{m-1} w+c^{m-2} L^{m-2} w+\ldots+c^{n} L^{n} w .
\end{aligned}
$$


Thus, the normality of the cone $K$ leads to

$$
\begin{aligned}
\left\|u_{m}-u_{n}\right\| & \leq n_{K}\left(c^{m-1}\left\|L^{m-1} w\right\|+c^{m-2}\left\|L^{m-2} w\right\|+\ldots+c^{n}\left\|L^{n} w\right\|\right) \\
& =n_{K}\left(S_{m-1}-S_{n-1}\right),
\end{aligned}
$$

where $S_{n}=\sum_{k=0}^{k=n} c^{k}\left\|L^{k} w\right\|$.

Since $w \succ 0_{E}$, we obtain

$$
\lim _{n \rightarrow+\infty} \sqrt[n]{\left\|c^{n} L^{n} w\right\|}=\lim _{n \rightarrow+\infty} \sqrt[n]{\left\|c^{n} L^{n} w\right\|} \leq c \lim _{n \rightarrow+\infty} \sqrt[n]{\|w\|} \sqrt[n]{\left\|L^{n}\right\|}=\operatorname{cr}(L)<1
$$

that is $\left(S_{n}\right)$ converges and

$$
\lim _{n \rightarrow+\infty}\left\|u_{m}-u_{n}\right\| \leq \lim _{n \rightarrow+\infty}\left\|S_{m-1}-S_{n-1}\right\|=0 .
$$

Therefore, the sequence $\left(u_{n}\right)_{n}$ is a Cauchy sequence and the completeness of $E$ leads to $\lim _{n \rightarrow+\infty} u_{n}=$ $u \in E$. Finally, since the mapping $|\cdot|$ is continuous and $L \in \mathcal{L}(E)$, the inequality in Condition 3 of Theorem 3.2 makes of $T$ a continuous mapping. Passing to the limit in $u_{n+1}=T u_{n}+v$, we obtain $u=T u+v$.

Step 2. We have proved in the above step that $(I-T)^{-1}$ exists. So, the purpose of this step, is to prove that $(I-T)^{-1}$ is continuous. To this aim, let $u, v \in E$ and set $x=(I-A)^{-1} u, y=(I-A)^{-1} v$. We have

$$
\begin{aligned}
|u-v| & =|(I-T) x-(I-T) y| \\
& =|(x-y)-(T x-T y)| \\
& \succeq_{K}|x-y|-|T x-T y| \\
& \succeq_{K}|x-y|-c L(|x-y|) \\
& =(I-c L)(|x-y|) \\
& =(I-c L)\left(\left|(I-T)^{-1} u-(I-T)^{-1} v\right|\right),
\end{aligned}
$$

leading to

$$
\left|(I-T)^{-1} u-(I-A)^{-1} v\right| \preceq_{K}(I-c L)^{-1}(|u-v|) .
$$

Taking in account the normality of the cone $K$ and the continuity of the mapping $|\cdot|$, we obtain from (3.2)

$$
\begin{aligned}
\left\|(I-T)^{-1} u-(I-T)^{-1} v\right\| & \leq n_{K}\left\|(I-T)^{-1} u-(I-A)^{-1} v \mid\right\| \\
& \leq n_{K}^{2}\left\|(I-c L)^{-1}(|u-v|)\right\| \\
& \leq n_{K}^{2}\left\|(I-c L)^{-1}\right\|\||u-v|\| \\
& \leq n_{K}^{2} \eta\left\|(I-c L)^{-1}\right\|\|u-v\| .
\end{aligned}
$$

Hence the continuity of $(I-T)^{-1}$ is proved.

Step 3. In this last step, we prove that the mapping $S+T$ has a fixed point in $\Omega$. To this aim, consider the mapping $\Phi=(I-T)^{-1} S: \Omega \rightarrow E$ and notice that $\Phi$ is compact. Let $v \in \Phi(\Omega)$, there exists $u \in \Omega$ 
such that $v=\Phi(u)=(I-T)^{-1} S(u)$. In an other manner, we have $v=S(u)+T(v)$ and Condition 1 leads to $v \in \Omega$. Thus, Theorem 2.11 guarantees existence of a fixed point $x \in \Omega$ for the mapping $\Phi$ and clearly, $x=S x+T x$. This ends the proof.

Remark 3.3. Theorem 3.2 holds true if replace Condition 1 by that of Burton, see [5],

$$
x=B x+A y, y \in M \Longrightarrow x \in M .
$$

Remark 3.4. A mapping $T: E \longrightarrow E$ satisfying Condition 3 of Theorem 3.2 is not necessarely a contraction, as an example we quote the mapping $T: C([0,1]) \longrightarrow C([0,1])$ defined by

$$
T u(x)=\frac{7 \pi^{2}}{4} \int_{0}^{1} J(x, s) \sqrt{1+|u(s)|} d s
$$

where for $x, s \in[0,1]$

$$
J(x, s)=\left\{\begin{array}{l}
x(1-s), \text { if } x \leq s \\
s(1-x), \text { if } s \leq x .
\end{array}\right.
$$

We have

$$
|T u(x)-T v(x)| \leq \frac{7 \pi^{2}}{8} L(|u-v|)(x),
$$

where $L u(x)=\int_{0}^{1} J(x, s) u(s) d s$ and $r(L)=\frac{1}{\pi^{2}}$.

The corresponding alternative to Theorem 3.2 consists in the following result.

Theorem 3.5. Assume that the cone $K$ is normal and minihedral and let $S, T: E \rightarrow E$ be two mappings such that $S$ is completely continuous and T satisfies condition 3 in Theorem 3.2. Then either

1. $S+T$ has a fixed point, or

2. the set $\left\{x \in E: x=\lambda T\left(\frac{x}{\lambda}\right)+\lambda S(x) \lambda \in(0,1)\right\}$ is unbounded.

Proof. Noticing that $x \in E$ is a fixed point of $S+T$ if and only if $x$ is a fixed point of $(I-T)^{-1} S$, we see that Theorem 3.5 follows from a direct application of Schaefer's theorem for the mapping $(I-T)^{-1} S$.

\section{Existence of solution for an integral equation}

We are concerned in this section by existence of solutions in $B C(\mathbb{R})$ to the integral equation

$$
u(x)=\int_{-\infty}^{+\infty} G_{1}(x, s) f_{1}(s, u(s)) d s+\int_{-\infty}^{+\infty} G_{2}(x, s) f_{2}(s, u(s)) d s,
$$

where $B C(\mathbb{R})$ denotes the set of all continuous bounded reel functions defined on $\mathbb{R}$ and for $i=1,2$, $G_{i}, f_{i}: \mathbb{R}^{2} \rightarrow \mathbb{R}$ are continuous functions. Throughout, we let $\phi: \mathbb{R} \rightarrow \mathbb{R}^{+}$be a continuous function 
and we let for $i=1,2, \rho_{i}$ be a nonnegative constant with $\rho_{2} \leq 1$ and $\alpha_{i}, \beta_{i}, \gamma_{i}: \mathbb{R} \rightarrow \mathbb{R}^{+}$be measurable functions such that

$$
\begin{aligned}
& \left\{\begin{array}{l}
\int_{-\infty}^{+\infty} \phi(s) \gamma_{2}(s) d s<\infty \text { and for } i=1,2 \\
\int_{-\infty}^{+\infty} \alpha_{i}(s) \gamma_{i}(s) d s<\infty, \int_{-\infty}^{+\infty} \beta_{i}(s) \gamma_{i}(s) d s<\infty
\end{array}\right. \\
& \left\{\begin{array}{l}
\left|G_{1}(x, s)-G_{1}(y, s)\right| \leq \gamma_{1}(t)|x-y| \text { for all } s, x, y \in \mathbb{R}, \\
\int_{-\infty}^{+\infty}\left|G_{1}(0, s)\right| \alpha_{1}(s) d s<\infty, \int_{-\infty}^{+\infty}\left|G_{1}(0, s)\right| \beta_{1}(s) d s<\infty \\
\lim _{|x| \rightarrow \infty} \int_{-\infty}^{+\infty}\left|G_{1}(x, s)\right| \alpha_{1}(s) d s=\lim _{|x| \rightarrow \infty} \int_{-\infty}^{+\infty}\left|G_{1}(x, s)\right| \beta_{1}(s) d s=0 \\
0 \leq G_{2}(x, t) \leq \gamma_{2}(t) \text { for all } x, t \in \mathbb{R}, \\
G_{2}(t, s) \phi(s)>0 \text { for all } t, s \in[\xi, \eta] \subset \mathbb{R} .
\end{array}\right. \\
& \left\{\begin{array}{l}
\left|f_{1}(s, u)\right| \leq \alpha_{1}(s)+\beta_{1}(s)|u|^{\rho_{1}} \text { for all } s, u \in \mathbb{R}, \\
\left|f_{2}(s, u)\right| \leq \gamma(s)+\delta(s)|u|^{\rho_{2}} \text { for all } s, u \in \mathbb{R} \text { and } \\
\left|f_{2}(s, u)-f_{2}(s, v)\right| \leq c \phi(s)|u-v| \text { for all } s, u, v \in \mathbb{R}, \\
\text { with } c \geq 0
\end{array}\right.
\end{aligned}
$$

Let $K$ denote the cone of nonnegative functions in $B C(\mathbb{R})$. Clearly, the cone $K$ is normal with constant 1 and $K$ is minihedral where for $u \in B C(\mathbb{R})$

$$
u^{+}(x)=\max (u(x), 0), u^{-}(x)=\max (-u(x), 0) \text { and }|u|(x)=u^{+}(x)+u^{-}(x) .
$$

Lemma 4.1. Assume that Hypotheses (4.2) and (4.3) hold and let for $u \in B C(\mathbb{R}), \operatorname{Lu}(x)=\int_{-\infty}^{+\infty}$ $G_{2}(x, s) \phi(s) u(s) d s$. Then $L$ is a linear operator belonging to $\mathcal{L}_{K}(B C(\mathbb{R}))$ with $r(L)>0$.

Proof. For all $R>0$ and $u \in B C(\mathbb{R})$ with $\|u\|_{\infty} \leq R$, we obtain from Hypotheses (4.2) and (4.3) the following estimates.

$$
\begin{aligned}
& |L u(x)| \leq \int_{-\infty}^{+\infty} G_{2}(x, s) \phi(s)|u(s)| d s \leq\left(\int_{-\infty}^{+\infty} \gamma_{2}(s) \phi(s) d s\right)\|u\|_{\infty}<\infty \\
& |L u(x)-L u(y)| \leq\left(\int_{-\infty}^{+\infty}\left|G_{2}(x, s)-G_{2}(y, s)\right| \phi(s) d s\right)\|u\|_{\infty} .
\end{aligned}
$$

Estimate (4.11) show that $L u(x)$ is defined for all $x \in \mathbb{R}$ and since $G_{2}$ is continuous and $G_{2}(x, s) \leq$ $\gamma_{2}(s)$ for all $x, s \in \mathbb{R}$, Estimates (4.11) and (4.6) combined with Lebesgue's dominated convergence theorem, lead to $L u \in C(\mathbb{R})$. Because that (4.5) provide a uniform bound, we conclude that $L u \in$ $B C(\mathbb{R})$ and $L \in \mathcal{L}(B C(\mathbb{R}))$. Clearly $L \in \mathcal{L}_{K}(B C(\mathbb{R}))$, let us prove that $r(L)>0$.

Let $u_{0}: \mathbb{R} \rightarrow[0,+\infty)$ be the function defined by

$$
\begin{aligned}
& u_{0}(t)=\left\{\begin{array}{l}
0, \text { if } t \in(-\infty, \xi] \\
\frac{4}{\eta-\xi}(t-\xi), \text { if } t \in\left[\xi, \frac{3 \xi+\eta}{4}\right] \\
1, \text { if } t \in\left[\frac{3 \xi+\eta}{4}, \frac{\xi+3 \eta}{4}\right] \\
\frac{4}{\eta-\xi}(\eta-t), \text { if } t \in\left[\frac{\xi+3 \eta}{4}, \eta\right] \\
0, \text { if } t \in[\eta,+\infty)
\end{array}\right. \\
& G_{0}=\min \left\{G_{2}(t, s) \phi(s): t, s \in[\xi, \eta]\right\} \\
& \theta_{0}=\int_{\xi}^{\eta} u_{0}(s) d s=\frac{3(\eta-\xi) G_{0}}{4} .
\end{aligned}
$$


We have

$$
\begin{aligned}
& L u_{0}(x) \geq 0=\theta_{0} u_{0}(x) \text { for } x \in(-\infty, \xi] \cup[\eta,+\infty) \text { and } \\
& L u_{0}(x) \geq \int_{\xi}^{\eta} G_{2}(x, s) \phi(s) u_{0}(x) d s \geq \theta_{0} \geq \theta_{0} u_{0}(x) \text { for } x \in[\xi, \eta] .
\end{aligned}
$$

Leading to $\left(\theta_{0}\right)^{-1} L u_{0} \geq u_{0}$, then we conclude by Lemma 3.1 that $r(L) \geq \theta_{0}>0$. The proof is complete.

The statement of the main result of this section needs to introduce the following notations:

$$
\begin{gathered}
\Gamma_{11}=\sup _{x \in \mathbb{R}} \int_{-\infty}^{+\infty}\left|G_{1}(x, s)\right| \alpha_{1}(s) d s, \quad \Gamma_{12}=\sup _{x \in \mathbb{R}} \int_{-\infty}^{+\infty}\left|G_{1}(x, s)\right| \beta_{1}(s) d s, \\
\Gamma_{21}=\sup _{x \in \mathbb{R}} \int_{-\infty}^{+\infty} G_{2}(x, s) \alpha_{2}(s) d s, \quad \Gamma_{22}=\sup _{x \in \mathbb{R}} \int_{-\infty}^{+\infty} G_{2}(x, s) \beta_{2}(s) d s .
\end{gathered}
$$

Theorem 4.2. Assume that Hypotheses (4.2)-(4.4) hold,

$$
\left\{\begin{array}{l}
\text { cr }(L)<1 \text { and there exists } \widetilde{R}>0 \text { such that } \\
\Gamma_{11}+\Gamma_{21}+\Gamma_{12}(\widetilde{R})^{\rho_{1}}+\Gamma_{22}(\widetilde{R})^{\rho_{2}} \leq \widetilde{R}
\end{array}\right.
$$

Then the integral equation admit a solution in the ball $B(0, \widetilde{R})$ of $B C(\mathbb{R})$.

Proof. Set for all $u \in B C(\mathbb{R})$,

$$
\begin{aligned}
& T_{1} u(x)=\int_{-\infty}^{+\infty} G_{1}(x, s) f_{1}(s, u(s)) d s \\
& T_{2} u(x)=\int_{-\infty}^{+\infty} G_{2}(x, s) f_{2}(s, u(s)) d s .
\end{aligned}
$$

For all $R>0$ and $u \in B C(\mathbb{R})$ with $\|u\|_{\infty} \leq R$, we obtain from Hypotheses (4.2)-(4.4) the following estimates.

$$
\begin{aligned}
& \left|T_{1} u(x)\right| \leq \int_{-\infty}^{+\infty}\left|G_{1}(x, s)\right|\left|f_{1}(s, u(s))\right| d s \\
& \leq \int_{-\infty}^{+\infty}\left|G_{1}(x, s)\right| \alpha_{1}(s) d s+R^{\rho_{1}} \int_{-\infty}^{+\infty}\left|G_{1}(x, s)\right| \beta_{1}(s) d s \\
& \leq|x|\left(\int_{-\infty}^{+\infty} \gamma_{1}(s) \alpha_{1}(s) d s+R^{\rho_{1}} \int_{-\infty}^{+\infty} \gamma_{1}(s) \beta_{1}(s) d s\right) \\
& +\int_{-\infty}^{+\infty}\left|G_{1}(0, s)\right| \alpha_{1}(s) d s+R^{\rho_{1}} \int_{-\infty}^{+\infty}\left|G_{1}(0, s)\right| \beta_{1}(s) d s<\infty \text {. } \\
& \left|T_{1} u(x)-T_{1} u(y)\right| \leq \int_{-\infty}^{+\infty}\left|G_{1}(x, s)-G_{1}(y, s)\right|\left|f_{1}(s, u(s))\right| d s \\
& \leq \int_{-\infty}^{+\infty} \gamma_{1}(s)\left|f_{1}(s, u(s))\right| d s|x-y| \\
& \leq\left(\int_{-\infty}^{+\infty} \gamma_{1}(s) \alpha_{1}(s) d s+R^{\rho_{1}} \int_{-\infty}^{+\infty} \gamma_{1}(s) \beta_{1}(s) d s\right)|x-y| \\
& \lim _{|x| \rightarrow \infty}\left|T_{1} u(x)\right| \leq \lim _{|x| \rightarrow \infty} \int_{-\infty}^{+\infty}\left|G_{1}(x, s)\right| \alpha_{1}(s) d s+R^{\rho_{1}} \lim _{|x| \rightarrow \infty} \int_{-\infty}^{+\infty}\left|G_{1}(x, s)\right| \beta_{1}(s) d s=0 \\
& \left|T_{2} u(x)\right| \leq \int_{-\infty}^{+\infty} G_{2}(x, s)\left|f_{2}(s, u(s))\right| d s \\
& \leq \int_{-\infty}^{+\infty} \gamma_{2}(s) \alpha_{2}(s) d s+R^{\rho_{2}} \int_{-\infty}^{+\infty} \gamma_{2}(s) \beta_{2}(s) d s<\infty \\
& \left|T_{2} u(x)-T_{2} u(y)\right| \leq \int_{-\infty}^{+\infty}\left|G_{2}(x, s)-G_{2}(y, s)\right|\left|f_{2}(s, u(s))\right| d s \\
& \leq \int_{-\infty}^{+\infty}\left|G_{2}(x, s)-G_{2}(y, s)\right| \alpha_{2}(s) d s+R^{\rho_{2}} \int_{-\infty}^{+\infty}\left|G_{2}(x, s)-G_{2}(y, s)\right| \beta_{2}(s) d s,
\end{aligned}
$$

Estimates (4.7) and (4.10) show respectively that for all $u \in B C(\mathbb{R})$ and $x \in \mathbb{R}, T_{1} u(x)$ and $T_{2} u(x)$ are defined. From Estimates (4.8) and (4.9), we see that $T_{1} u \in B C(\mathbb{R})$ and since $G_{2}$ is continuous and 
$G_{2}(x, s) \leq \gamma_{2}(s)$ for all $x, s \in \mathbb{R}$, Estimate (4.11) combined with Lebesgue's dominated convergence theorem, leads to $T_{2} u \in C(\mathbb{R})$. Because that (4.10) provide a uniform bound, we conclude that $T_{2} u \in$ $B C(\mathbb{R})$. Therefore, we have proved that $T_{1}$ and $T_{2}$ define self mappings on $B C(\mathbb{R})$.

Now, for $u, v \in B C(\mathbb{R})$, we have

$$
\begin{aligned}
\left|T_{2} u(x)-T_{2} v(x)\right| & \leq \int_{-\infty}^{+\infty} G_{2}(x, s)\left|f_{2}(s, u(s))-f_{2}(s, v(s))\right| d s \\
\leq & c \int_{-\infty}^{+\infty} G_{2}(x, s) \phi(s)|u(s)-v(s)| d s \\
& =c L|u-v|(x) .
\end{aligned}
$$

Thus, Condition 3 in Theorem 3.2 is satisfied.

In order to demonstrate that $T_{1}$ is continuous, let $\left(u_{n}\right)$ be a sequence in $B C(\mathbb{R})$ converging to $u$ and suppose $\left(u_{n}\right) \subset B\left(0, R_{0}\right)$ for some $R_{0}>0$. Let $\epsilon>0$. Since

$$
\left|T_{1} u_{n}(x)-T_{1} u(x)\right| \leq 2 \int_{-\infty}^{+\infty}\left|G_{1}(x, s)\right| \alpha_{1}(s) d s+2 R_{0}^{\rho_{1}} \int_{-\infty}^{+\infty}\left|G_{1}(x, s)\right| \beta_{1}(s) d s
$$

and

$$
\lim _{|x| \rightarrow \infty}\left(\int_{-\infty}^{+\infty}\left|G_{1}(x, s)\right| \alpha_{1}(s) d s+2 R_{0}^{\rho_{1}} \int_{-\infty}^{+\infty}\left|G_{1}(x, s)\right| \beta_{1}(s) d s\right)=0,
$$

there is $A>0$ such that

$$
\sup _{|x| \geq A}\left|T_{1} u_{n}(x)-T_{1} u(x)\right| \leq \epsilon .
$$

Then, for all $x \in[-A, A]$ we have

$$
\begin{gathered}
\left|T_{1} u_{n}(x)-T_{1} u(x)\right| \leq A \int_{-\infty}^{+\infty} \gamma_{1}(s)\left|f_{1}\left(s, u_{n}(s)\right)-f_{1}(s, u(s))\right| d s \\
+\int_{-\infty}^{+\infty} G_{1}(0, s)\left|f_{1}\left(s, u_{n}(s)\right)-f_{1}(s, u(s))\right| d s
\end{gathered}
$$

and because that

$$
\begin{aligned}
& \left|f_{1}\left(s, u_{n}(s)\right)-f_{1}(s, u(s))\right| \rightarrow 0 \text { as } n \rightarrow \infty \text { for all } s \in \mathbb{R}, \\
& \left|f_{1}\left(s, u_{n}(s)\right)-f_{1}(s, u(s))\right| \leq 2 \alpha_{1}(s)+2 R_{0}^{\rho_{1}} \beta_{1}(s), \\
& \int_{-\infty}^{+\infty} \gamma_{1}(s) \alpha_{1}(s) d s<\infty, \int_{-\infty}^{+\infty} \gamma_{1}(s) \beta_{1}(s) d s<\infty \\
& \int_{-\infty}^{+\infty}\left|G_{1}(0, s)\right| \alpha_{1}(s) d s<\infty, \text { and } \int_{-\infty}^{+\infty}\left|G_{1}(0, s)\right| \beta_{1}(s) d s<\infty,
\end{aligned}
$$

we conclude by Lebesgue's dominated convergence theorem that

$$
\sup _{|x| \leq A}\left|T_{1} u_{n}(x)-T_{1} u(x)\right| \leq \epsilon \text { for } n \text { large. }
$$

Therefore, we have proved that

$$
\lim _{n \rightarrow \infty}\left\|T_{1} u_{n}-T_{1} u\right\|_{\infty}=\lim _{n \rightarrow \infty}\left(\sup _{x \in \mathbb{R}}\left|T_{1} u_{n}(x)-T_{1} u(x)\right|\right)=0
$$

and $T_{1}$ is continuous.

Estimates (4.8) and (4.9) show respectively that for all $R>0, T_{1}(B(0, R))$ is locally equicontinuous and $T_{1}(B(0, R))$ is equiconvergent at $\pm \infty$. 
Being equiconvergent, there exists $A>0$ such that

$$
\begin{aligned}
& \sup _{\|u\|_{\infty} \leq R}\left\|T_{1} u\right\|_{\infty}=\sup _{\|u\|_{\infty} \leq R}\left(\sup _{|x| \leq A}\left|T_{1} u(x)\right|\right) \\
& \leq A \int_{-\infty}^{+\infty} \gamma_{1}(s)\left(\alpha_{1}(s)+R^{\rho_{1}} \beta_{1}(s)\right) d s+\int_{-\infty}^{+\infty}\left|G_{1}(0, s)\right|\left(\alpha_{1}(s)+R^{\rho_{1}} \beta_{1}(s)\right) d s,
\end{aligned}
$$

proving that $T_{1}(B(0, R))$ is bounded.

Thus, Corduneanu's compactness criterion ([7], p. 62) guarantees that $T_{1}(B(0, R))$ is relatively compact in $C_{0}(\mathbb{R})$, then in $B C(\mathbb{R})$, proving that $T_{1}$ is completely continuous.

At the end, it is easy to see that $\left(T_{1}+T_{2}\right)(B(0, \widetilde{R})) \subset B(0, \widetilde{R})$ and we conclude by Theorem 3.2, that Equation (4.1) admits a solution in the ball $B(0, \widetilde{R})$ of $B C(\mathbb{R})$.

\section{Comments and open questions.}

1) If $S=0$, we deduce from Theorem 3.2 and its proof (see Step 1) the following corollary which provides a variant of Banach contraction principle in ordered Banach spaces:

Corollary 4.3. Assume that the cone $K$ is normal and minihedral and let $\Omega$ be a closed convex nonempty subset of $E$. If

$$
|T u-T v| \preceq_{K} c L(|u-v|) \text { for all } u, v \in E,
$$

where $L \in \mathcal{L}_{K}(E)$ with $r(L)>0$ and $c \in[0,1 / r(L))$, then $T$ has a unique fixed point in $\Omega$.

From Browder's fixed point theorem for nonexpansive mappings arise the following question: does Corollary 4.3 hold if $\operatorname{cr}(L)=1$ and $E$ is uniformly convex ?

2) From Theorem 2.1 in [22] arise the following question: does Theorem 3.2 hold true if the Condition 2 is replaced by $S(\Omega)$ is relatively weakly compact ?

3) It is interesting to consider the case where the mapping $T$ lies in $\mathcal{L}(E)$, as it is done in $[2,3,9,10]$.

4) Since there are many results in the literature considering the case of nonlinear contraction, it seems to be interesting to investigate the case where the operator $L$ is nonlinear.

Acknowledgement. The author is thankful to the anonymous referee for his deep and careful reading of the manuscript and for all his comments and suggestions, which led to a substantial improvement of the original manuscript.

\section{References}

[1] R. P. Agarwal, D. O'Regan and M. A. Taoudi, Browder-Krasnoselskii-type fixed point theorems in Banach spaces, Fixed Point Theory Appl., Volume 2010, Article ID 243716, 20 pages.

[2] C. S. Barroso, Krasnosel'skii's fixed point theorem for weakly continuous maps, Nonlinear Anal. 55 (2003), 25-31.

[3] C. S. Barroso and E. V. Teixeira, A topological and geometric approach to fixed points results for sum of operators and applications, Nonlinear Anal. 60 (2005), 625-650.

[4] M. Berzig, S. Chandok and M. S. Khan, Generalized Krasnoselskii fixed point theorem involving auxiliary functions in bimetric spaces and application to two-point boundary value problem, Appl. Math. Comput. 248 (2014) 323-327. 
[5] T. A. Burton, A fixed-point theorem of Krasnoselskii, Appl. Math. Lett., 11 (1998), 85-88.

[6] T. A. Burton and C. Kirk, A fixed point theorem of Krasnoselskii-Schaefer type, Math. Nachr., 189 (1998), $23-31$.

[7] C. Corduneanu, Integral Equations and Stability of Feedback Systems, Academic Press, New York, 1973.

[8] K. Deimling, Nonlinear Functional Analysis, Springer-Verlag, 1985.

[9] B. C. Dhage, On a fixed point theorem of Krasnoselskii-Schaefer type, Electron. J. Qual. Theory Differ. Equ., 2002 No. 6, 1-9.

[10] B. C. Dhage, Some variants of two basic hybrid fixed point theorems of Krasnoselskii with applications, Nonlinear Stud., 25 (2018), 559-573.

[11] J. Garcia-Falset, K. Latrach, E. Moreno-Gálvez, M. A. Taoudid, Schaefer-Krasnoselskii fixed point theorems using a usual measure of weak noncompactness, J. Differential Equations 252 (2012) 3436-3452.

[12] J. Garcia-Falset and K. Latrach, Krasnoselskii-type fixed-point theorems for weakly sequentially continuous mappings, Bull. London Math. Soc. 44 (2012) 25-38.

[13] D. Guo and V. Lakshmikantaham, Nonlinear Problems in Abstract Cones, Academic Press, San Diego, 1988.

[14] M. A. Krasnosel'skii, Some problems of nonlinear analysis, Amer. Math. Soc. Transl. Ser. 2, 10 (1958), 345-409.

[15] Y. Liu and Z. Li, Krasnoselskii type fixed point theorems and applications, Proc. Amer. Math. Soc., 136 (2008), 1213-1220.

[16] W. A. J. Luxemburg and A. C. Zaanen, Riesz spaces I, North-Holland publishing company, 1971.

[17] W. R. Melvin, Some extensions of the Krasnoselskii fixed point theorems, J. Differential Equations, 11 (1972), 335-348.

[18] S. Park, Generalizations of the Krasnoselskii fixed point theorem, Nonlinear Anal., 67 (2007) 3401-3410.

[19] V. M. Sehgal and S. P. Singh, On a fixed point theorem of Krasnoselskii for locally convex spaces, Pacific J. Math. 62 (1976), 561-567.

[20] D. R. Smart, Fixed point theorems, Cambrige university press, 1974.

[21] T. Xiang and S. G. Georgiev, Noncompact-type Krasnoselskii fixed-point theorems and their applications, Math. Methods Appl. Sci., 2015, DOI: 10.1002/mma.3525.

[22] M. A. Taoudi, Krasnosel'skii type fixed point theorems under weak topology features, Nonlinear Anal., 72 (2010), 478-482.

[23] K. Yoshida, Functional Analysis, Springer-Verlag, 1980. 